\title{
Inhibition of miR-23a increases the sensitivity of lung cancer stem cells to erlotinib through PTEN/PI3K/Akt pathway
}

\author{
ZHIJUN HAN ${ }^{1,2}$, XIAOYUN ZHOU ${ }^{1,2}$, SHANQING LI ${ }^{1,2}$, YINGZHI QIN $^{1,2}$, YEYE CHEN $^{1,2}$ and HONGSHENG LIU ${ }^{1,2}$ \\ ${ }^{1}$ Department of Thoracic Surgery, Peking Union Medical College Hospital (PUMCH); \\ ${ }^{2}$ Chinese Academy of Medical Sciences and Peking Union Medical College (CAMS \& PUMC), Beijing 100730, P.R. China
}

Received February 22, 2017; Accepted August 2, 2017

DOI: $10.3892 /$ or.2017.5938

\begin{abstract}
Epidermal growth factor receptor-targeted tyrosine kinase inhibitors (EGFR-TKIs) have become first-line drugs used for non-small cell lung cancer (NSCLC) treatment. However, drug resistance to EGFR-TKIs will be developed inevitably due to the repeated use of these drugs. In the present study, we isolated cancer stem cells (CSCs) from the PC9 NSCLC cell line. We then observed that the PC9 CSCs showed significant resistance to erlotinib compared with the PC9 non-CSCs. Erlotinib failed to suppress the phosphorylation of PI3K and AKT in PC9 CSCs, although the EGFR was inhibited sufficiently. Mechanically, we observed aberrant upregulation of microRNA-23a (miR-23a) and downregulation of PTEN in PC9 CSCs compared to PC9 non-CSCs. Luciferase reporter assays proved that PTEN was the target of miR-23a in PC9 CSCs. Furthermore, knockdown of miR-23a enhanced the antitumor effect of erlotinib by increasing the expression of PTEN. In addition, transfection with miR-23a inhibitors promoted the erlotinib-dependent inhibition of PI3K/AKT pathway, thus, suppressing the proliferation and inducing apoptosis in PC9 CSCs. These results propose that upregulation of miR-23a is a potential mechanism associated with resistance to EGFR-TKIs in lung cancer stem cells. Inhibition of miR-23a serves as a novel therapeutic strategy to eliminate the EGFR-TKIs resistance of lung cancer stem cells.
\end{abstract}

\section{Introduction}

Non-small cell lung cancer (NSCLC) is a leading cause of cancer-related mortality worldwide. Overall 5-year survival rate of NSCLC is lower than $5 \%(1,2)$. For treatment of NSCLC, epidermal growth factor receptor-targeted tyrosine kinase inhibitors (EGFR-TKIs) such as gefitinib, erlotinib

Correspondence to: Dr Hongsheng Liu, Department of Thoracic Surgery, Peking Union Medical College Hospital, Shuaifuyuan, No. 1 Dongcheng District, Beijing 100730, P.R. China E-mail: bjhongshengliu@sina.com

Key words: cancer stem cells, miR-23a, erlotinib, PI3K/AKT, EGFR-TKIs and afatinib have become first-line drugs for the NSCLC patients harboring EGFR active mutations (3-5). However, the insensitive subgroup of lung cancer cells can survive under the EGFR-TKIs treatment. Thus, tumor recurrence and acquired drug resistance is inevitable due to the repeated use of EGFR-TKIs (6).

Recently, studies has demonstrated that cancer stem cells (CSCs), which are a small subgroup of cells in tumor possessing the ability of self-renewal, are capable of driving tumorigenesis. Accumulating evidence indicated that lung cancer stem cells are responsible for resistance to chemotherapeutics and EGFR-TKIs (7-9). CSCs have become the target for reversing the resistance of lung cancer to EGFR-TKIs.

MicroRNAs (miRNAs) are single-stranded RNAs with a length of 21-25 nucleotides. They regulate cancer initiation and progression by regulating the expression of cancer-related genes. miRNAs usually act as negative regulators of gene expression by binding to the mRNAs at the 3'untranslated region (3'UTR) (10,11). Accumulating evidence has indicated that aberrant miRNAs is responsible to cancer survival, development and drug resistance $(12,13)$. In CSCs, it is reported that miRNAs are usually dysregulated in various types of cancers. Correcting the disorder of miRNAs in CSCs has become an important strategy for cancer therapy (14-16). Previous research has identified CD133 as a molecular marker of lung cancer stem cells (17). In the present study, we found that the expression of miR-23a was significantly upregulated in CD133 positive PC9 CSCs. Inhibition of miR-23a increases the sensitivity of lung cancer stem cells to erlotinib by upregulating the PTEN expression.

\section{Materials and methods}

Cell culture and CSC isolation. The NSCLC cell line PC9 was purchased from the American Type Culture Collection (ATCC; Manassas, VA, USA) and was cultured in RPMI-1640 medium containing 10\% fetal bovine serum (FBS; Gibco, Waltham, MA, USA). For isolating CSCs of PC9 cell line, FACSVantage (BD FACSCalibur; BD Biosciences, San Diego, CA, USA) was used to identify and collect the CD133-positive PC9 cells (CD133-FITC antibody was purchased from Miltenyi Biotec $\mathrm{GmbH}$, Bergisch Gladbach, Germany). To estimate the purity of CSC population in PC9, flow cytometry was performed. 
Transfection. Negative control oligonucleotides (miR-NC), microRNA-23a mimics (miR-23a), miR-23a inhibitors (anti-miR-23a) and PTEN siRNA were purchased from Guangzhou RiboBio, Co., Ltd. (Guangzhou, China). For transfection, $50 \mathrm{pmol} / \mathrm{ml} \mathrm{miR}-\mathrm{NC}$, miR-23a, anti-miR-23a or PTEN siRNA were packaged with Lipofectamine 2000 (Invitrogen, Waltham, MA, USA). Then, PC9 CSCs were incubated with RNAs in serum-free medium (Gibco). Six hours later, the culture medium was changed to 10\% FBS RPMI-1640 medium for $24 \mathrm{~h}$.

Cell viability assay. PC9 CSCs $\left(5 \times 10^{3}\right)$ or PC 9 non-CSCs $\left(5 \times 10^{3}\right)$ were plated on 96-well plates with $200 \mu 1$ RPMI-1640 medium. Then, cells were transfected with RNAs followed by treating with EGFR-TKIs for $48 \mathrm{~h}$. After incubation, $20 \mu \mathrm{l}$ 3-(4,5-dimethylthiazol-2-yl)-2,5-diphenyltetrazolium bromide (MTT) $(5 \mathrm{mg} / \mathrm{ml}$; Sigma-Aldrich, St. Louis, MO, USA) was added into the medium for $4 \mathrm{~h}$. Subsequently, we replaced the culture medium with $150 \mu$ l dimethyl sulfoxide (DMSO) for 30 min. Cell viability of PC9 CSCs and PC9 non-CSCs was evaluated according to the absorbance at $570 \mathrm{~nm}$ using a microplate reader. Half maximal inhibitory concentration $\left(\mathrm{IC}_{50}\right)$ of EGFR-TKIs to PC9 CSCs and PC9 non-CSCs was calculated according to the results of cell viability assays.

Cell proliferation assays. $\mathrm{PC} 9 \mathrm{CSCs}\left(5 \times 10^{5}\right)$ or $\mathrm{PC} 9$ non-CSCs $\left(5 \times 10^{5}\right)$ were plated on 6 -well plates with $2 \mathrm{ml}$ RPMI-1640 medium. Then, cells were transfected with RNAs followed by treating with $0.04 \mu \mathrm{M}$ erlotinib for $48 \mathrm{~h}$. After incubation, ${ }^{3} \mathrm{H}-$ thymidine was added into the culture medium for $6 \mathrm{~h}$. Then, ${ }^{3} \mathrm{H}$-thymidine incorporation assays were performed to determine the cell proliferation of PC9 CSCs and PC9 non-CSCs.

Colony forming assay. PC9 CSCs and PC9 non-CSCs were seeded at a density of 50 cells $/ \mathrm{cm}^{2}(185$ cells/well $)$ in a 12-well plate format. Subsequently, cells were treated with $0.04 \mu \mathrm{M}$ erlotinib for 10 days. After treatment, cell colonies were stained with crystal violet/ethanol mixture and then were counted under a microscope. The proportion of colonies formed is shown as a percentage of its untreated control (18).

Reverse transcription-quantitative polymerase chain reaction $(R T-q P C R)$. Total RNA was extracted from the PC9 CSCs and PC9 non-CSCs using TRIzol reagent (Invitrogen). Expression of miR-23a, Oct4 and Sox 2 was measured by One-Step RT-qPCR with SYBR Premix Ex Taq (Takara Bio Inc., Otsu, Japan) according to the manufacturer's instructions. U6 snRNA was used as the internal control to calculate the relative expression of miR-23a. GAPDH was used as the internal control to calculate the relative expression of Oct4 and Sox 2. $2^{-\Delta \Delta C T}$ method was used to analyze the results of RT-qPCR (19).

Western blot analysis. Total proteins were extracted from the PC9 CSCs and PC9 non-CSCs using RIPA buffer (Cell Signaling Technology, Beverly, MA, USA) and then quantified by bicinchoninic acid assay kit (Beyotime Institute of Biotechnology, Haimen, China). Proteins were then separated by $12 \%$ sodium dodecyl sulfate polyacrylamide gel electrophoresis (SDS-PAGE) and were transferred onto a PVDF membrane (Millipore, Billerica, MA, USA). Membranes were then blocked in 5\% skim milk for $1 \mathrm{~h}$ at room temperature and then incubated overnight at $4{ }^{\circ} \mathrm{C}$ with the following antibodies: anti-PTEN, anti-EGFR, anti-phosphorylated EGFR, anti-PI3K, anti-phosphorylated PI3K, anti-AKT, anti-phosphorylated AKT, anti-pro-caspase-9, anti-cleaved caspase- 9 , anti-pro-caspase- 3 , anti-cleaved caspase- 3 and anti- $\beta$-actin (Cell Signaling Technology). After washing the membranes with Tris-buffered saline, they were incubated with goat anti-rabbit secondary antibody (Cell Signaling Technology) for $1 \mathrm{~h}$ at room temperature. The blots were visualized using an enhanced chemiluminescence detection kit (Pierce, Rockford, IL, USA).

Apoptosis analysis. PC9 CSCs $\left(5 \times 10^{5}\right)$ or PC9 non-CSCs $\left(5 \times 10^{5}\right)$ were plated on 6-well plates with $2 \mathrm{ml}$ RPMI-1640 medium. Then, cells were transfected with RNAs followed by treating with $0.04 \mu \mathrm{M}$ erlotinib for $48 \mathrm{~h}$. After incubation, cells were collected and were stained with PI and Annexin V (Sigma-Aldrich) for $20 \mathrm{~min}$ at room temperature. Subsequently, percentage of apoptotic cells was quantified using flow cytometry.

Luciferase reporter assay. 3'UTR of PTEN mRNA was amplified and inserted into pGL3 Luciferase reporter vectors (Promega, Madison, WI, USA) downstream of the luciferase gene. PC9 CSCs $\left(1 \times 10^{5}\right)$ were plated on 24-well plates with $500 \mu \mathrm{l}$ RPMI-1640 medium. Then, cells were transfected with $50 \mathrm{pmol} / \mathrm{ml} \mathrm{miR}-23 \mathrm{a}$ mimics, $2 \mu \mathrm{g} / \mathrm{ml}$ firefly luciferase reporters and $100 \mathrm{ng} / \mathrm{ml}$ Renilla luciferase pRL-TK vector (Promega) for $24 \mathrm{~h}$. After transfection, cells were collected and washed with phosphate-buffered saline (PBS). Luciferase reporter assays were then performed using the Dual-Luciferase reporter assay system (Promega) according to the manufacturer's protocol.

Statistical analysis. Quantitative data are presented as the mean $\pm \mathrm{SD}$. All of the experiments were repeated in triplicate. For comparison analysis, the statistical analysis was performed by the Student's t-test using SPSS 15.0 software (SPSS; Chicago, IL, USA). A value of $\mathrm{P}<0.05$ was considered to indicate a statistically significant difference.

\section{Results}

PC9 cancer stem cells show resistance to erlotinib. To study the effect of EGFR-TKIs on lung cancer stem cells, we isolated the CSCs population of PC9 using CD133 antibody. Our separated PC9 CSCs expressed high level of CD133, and CD133 negative PC9 cell population was considered as the non-CSCs (Fig. 1A). As Oct4 and Sox 2 were identified as the markers of CSCs (20), we detected their expression by using RT-qPCR analysis. As shown in Fig. 1B, the CD133 positive PC9 cell population expressed significantly higher level of Oct4 and Sox 2 compared to the PC9 non-CSCs. Notably, we found that PC9 CSCs showed significant resistance to erlotinib compared to the PC9 non-CSCs. IC $_{50}$ of erlotinib to PC9 CSCs is 12.6-fold higher than the PC9 non-CSCs (Fig. 1C). The results suggested that lung cancer stem cells are resistant to EGFR-TKIs-induced cell death. 
A
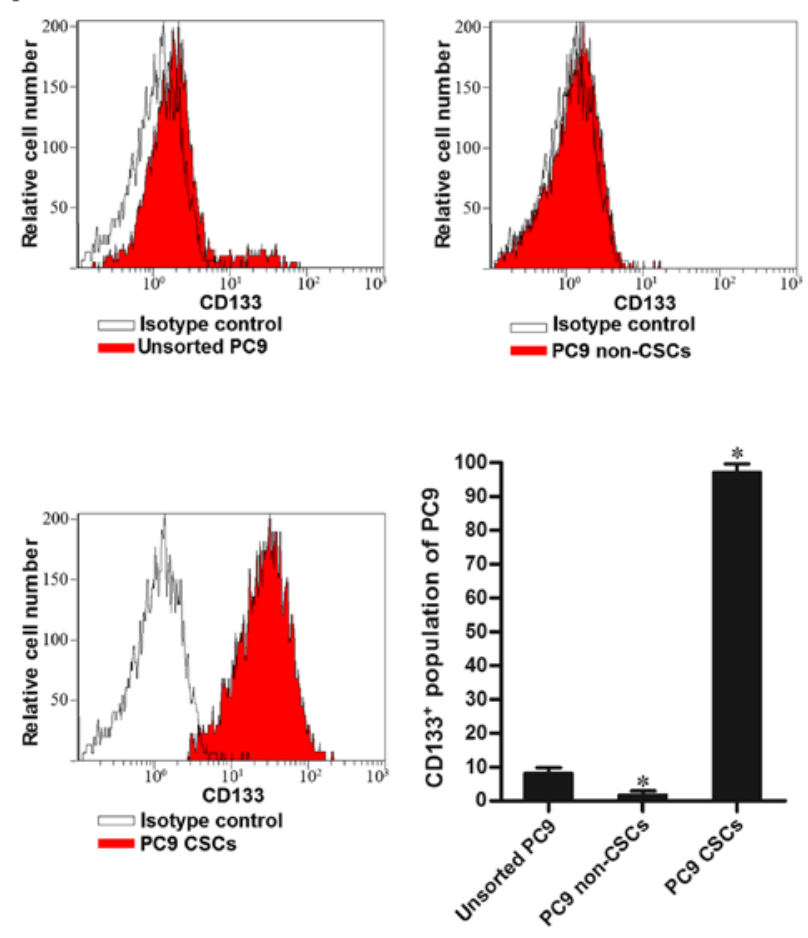

B
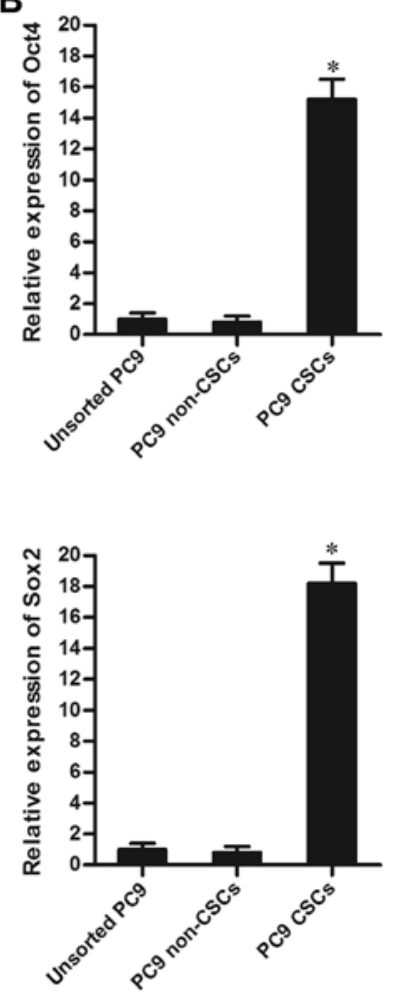

C
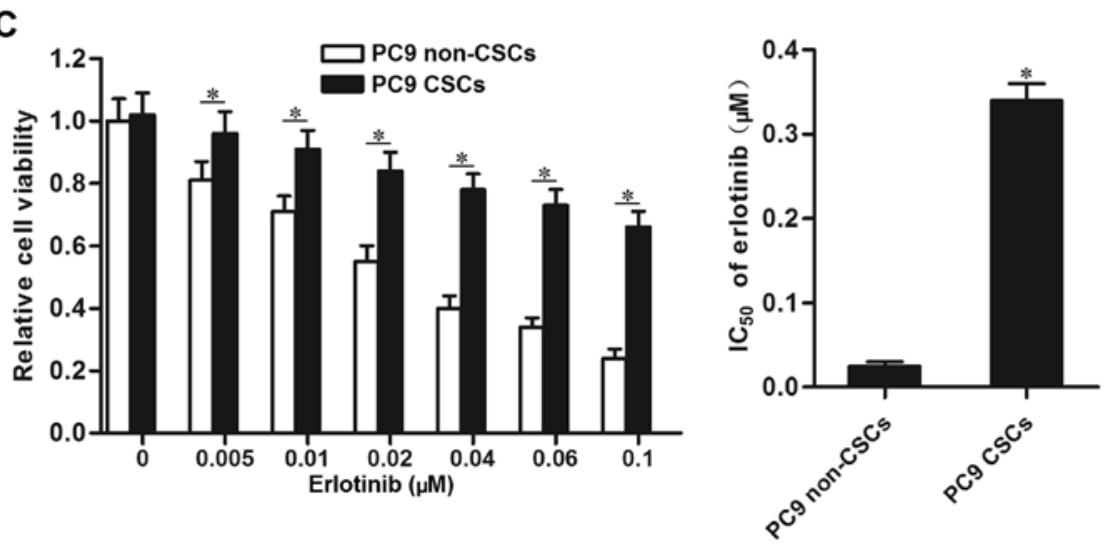

Figure 1. PC9 CSCs are resistant to erlotinib. (A) CD133 antibody was used to sort the CSC population in PC9 cell line. Purity of PC9 CSCs and PC9 non-CSCs was analyzed by flow cytometry. "P $<0.05$ vs. unsorted PC9 cells. (B) Expression of Oct4 and Sox 2 in PC9 CSCs and PC9 non-CSCs was detected by RT-qPCR. ${ }^{*} \mathrm{P}<0.05$ vs. PC9 non-CSCs. (C) Antitumor effect of erlotinib on PC9 CSCs and PC9 non-CSCs was evaluated by MTT assays. ${ }^{*} \mathrm{P}<0.05$. IC ${ }_{50}$ of erlotinib was compared between PC9-CSCs and PC9-non-CSCs. " $\mathrm{P}<0.05$ vs. PC9 non-CSCs.

Erlotinib fails to suppress the phosphorylation of PI3K and AKT in PC9 CSCs. Suppression of EGFR/PI3K/AKT pathway is the mechanism of EGFR-TKIs in killing the NSCLC cells (21). Consistent with this, phosphorylation of EGFR, PI3K and AKT was significantly inhibited in PC9 non-CSCs treated with erlotinib. However, in PC9 CSCs, we observed that erlotinib failed to suppress the activation of PI3K and AKT obviously, despite inhibiting the EGFR phosphorylation. High activation of PI3K and AKT promotes cell proliferation and inhibits apoptosis in cancer cells $(22,23)$. Since erlotinib failed to suppress the PI3K/AKT pathway in PC9 CSCs, the results of ${ }^{3} \mathrm{H}$-thymidine incorporation assays showed that PC9 CSCs still exhibited high proliferative activity even when they were treated with erlotinib (Fig. 2B). Similarly, the effect of erlotinib on suppressing the colony formation of PC9 CSCs was significantly less than the PC9
non-CSCs (Fig. 2C). Furthermore, PC9 CSCs showed obvious resistance to erlotinib-induced apoptosis rather than the PC9 non-CSCs (Fig. 2D). Taken together, we demonstrated that erlotinib failed to suppress the activation of PI3K and AKT in lung cancer stem cells.

PTEN is the target of miR-23a in PC9 cancer stem cells. To investigate the mechanism by which erlotinib failed to inhibit the PI3K/AKT pathway in PC9 CSCs, we detected the miRNA expression in PC9 cell line. We found that the expression of miR-23a was significantly upregulated in PC9 CSCs rather than PC9 non-CSCs (Fig. 3A). Furthermore, PTEN, which is an effective suppressor of PI3K and AKT (22), was downregulated in PC9 CSCs compared to the corresponding non-CSCs (Fig. 3B). Negative correlation between miR-23a and PTEN was observed. As the public miRNA TargetScan database (www.targetscan. 
A

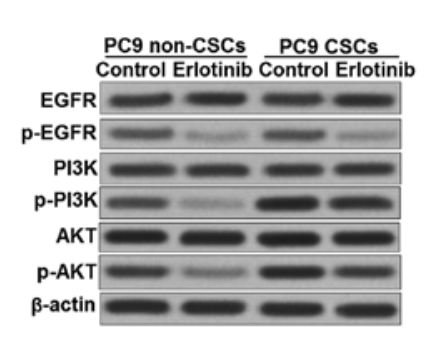

B

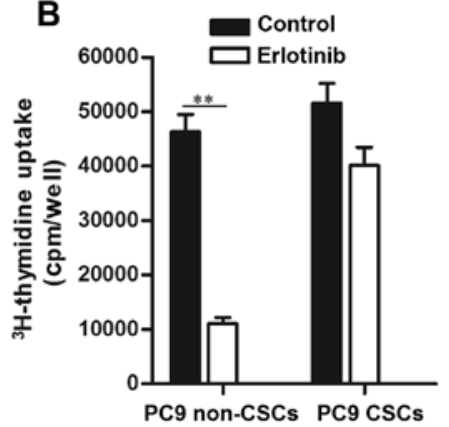

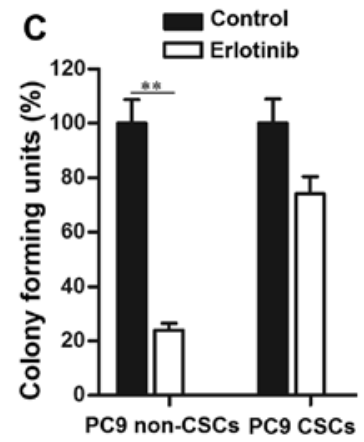

D

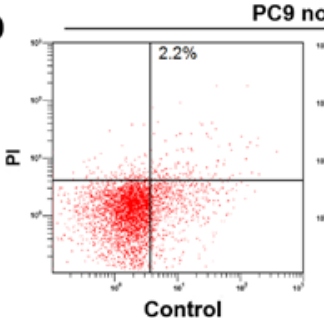

PC9 non-CSCs
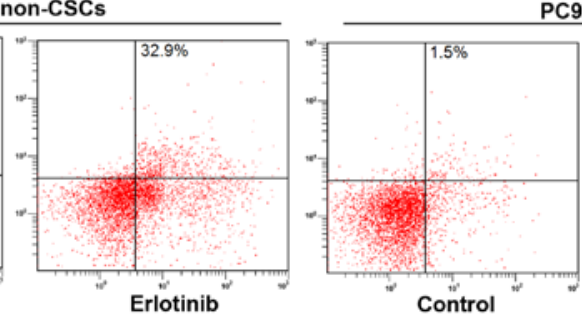

PC9 CSCs

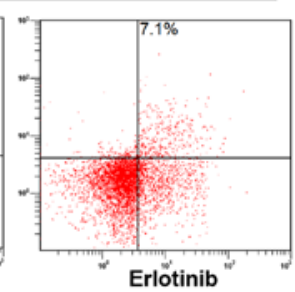

Annexin V

Figure 2. Erlotinib failed to suppress the phosphorylation of PI3K and AKT in PC9 CSCs. (A) Phosphorylation of EGFR, PI3K and AKT in PC9 non-CSCs and PC9 CSCs was detected by western blot analysis after they were treated with $0.04 \mu \mathrm{M}$ erlotinib. (B) Cell proliferation of PC9 non-CSCs and PC9 CSCs was measured by ${ }^{3} \mathrm{H}$-thymidine incorporation assays after they were treated with $0.04 \mu \mathrm{M}$ erlotinib. ${ }^{* *} \mathrm{P}<0.01$. (C) Colony formation of PC9 non-CSCs and PC9 CSCs was evaluated after they were treated with $0.04 \mu \mathrm{M}$ erlotinib. ${ }^{* * *} \mathrm{P}<0.01$. (D) Apoptosis of PC9 non-CSCs and PC9 CSCs was measured by flow cytometry after they were treated with $0.04 \mu \mathrm{M}$ erlotinib.

A

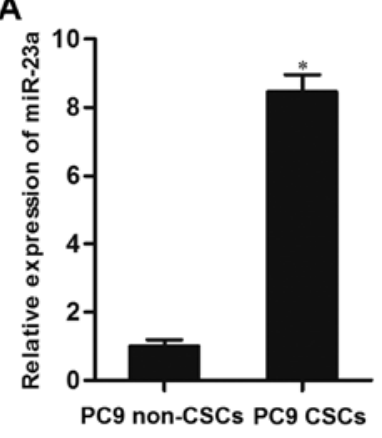

B

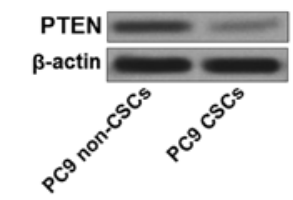

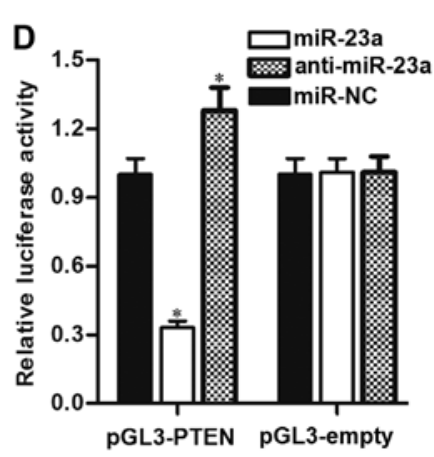

E

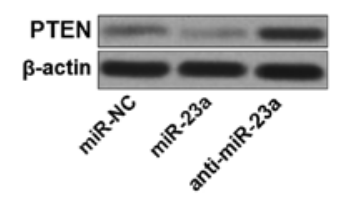

C

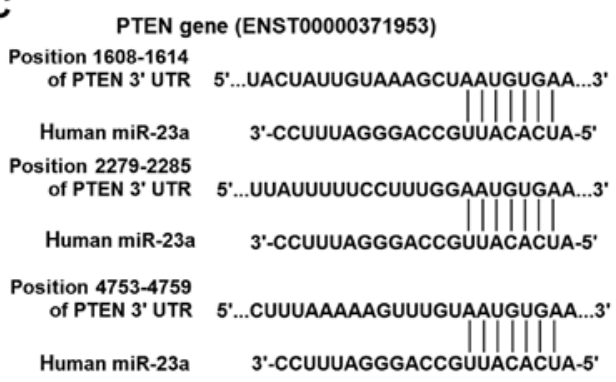

Figure 3. PTEN is the target of miR-23a. (A) Expression of miR-23a in PC9 CSCs and PC9 non-CSCs was detected by RT-qPCR. "P<0.05 vs. PC9 nonCSCs. (B) PTEN expression in PC9 CSCs and PC9 non-CSCs was measured by western blot analysis. (C) PTEN is predicted as the target of miR-23a by TargetScan databases. (D) miR-23a decreased the activities of luciferase in pGL3-PTEN plasmid. " $\mathrm{P}<0.05$ vs. miR-NC group. (E) Western blot analysis was performed to detect the expression of PTEN after the PC9 CSCs were transfected with miR-23a mimics or antisense oligonucleotides.

org) as well as miRanda database (http://www.microrna.org/) predicted that PTEN mRNA 3'UTR contained putative binding sites to miR-23a (Fig. 3C), we inferred that PTEN is the target of miR-23a, and overexpression of miR-23a in PC9 CSCs was responsible for their erlotinib resistance. To confirm the relationship between miR-23a and PTEN, we performed luciferase reporter assays. The results of these assays showed that transfection with miR-23a in PC9 CSCs significantly decreased the activities of luciferase in pGL3-PTEN plasmid. In contrast, introduction with anti-miR-23a was able to increase the activities of luciferase in pGL3-PTEN vector (Fig. 3D). These results implied the effect of miR-23a on regulating the PTEN gene. In addition, we observed that transfection with miR-23a decreased the protein level of PTEN, whereas the miR-23a antisense oligonucleotides (anti-miR-23a) significantly increased the expression level of PTEN in PC9 CSCs (Fig. 3E). Taken together, we proved that miR-23a regulated the expression of PTEN in lung cancer stem cells.

miR-23a antisense oligonucleotides resensitize PC9 CSCs to erlotinib by increasing the expression of PTEN. The preceding results demonstrated that miR-23a which was overexpressed in PC9 CSCs decreased the PTEN expression. We therefore investigated whether knockdown of miR-23a enhances the antitumor effect of erlotinib on PC9 CSCs. Results of western blot analysis illustrated that transfection with miR-23a antisense oligonucleotides (anti-miR-23a) significantly increased the expression of PTEN, whereas the PTEN siRNA abolished the effect of anti-miR-23a (Fig. 4A). Next, we found that miR-23a antisense oligonucleotides significantly enhanced the effect of erlotinib on killing PC9 CSCs. However, co-transfection with PETN siRNA obviously impaired the effect of anti-miR-23a (Fig. 4B). These results 
A

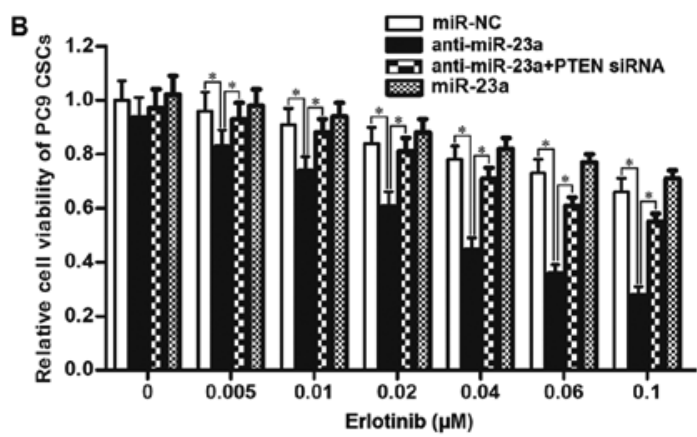

C

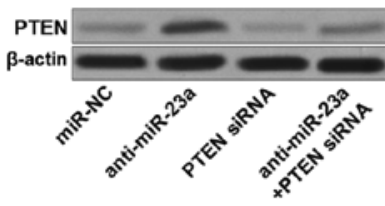

Erlotinib ( $\mu \mathrm{M})$

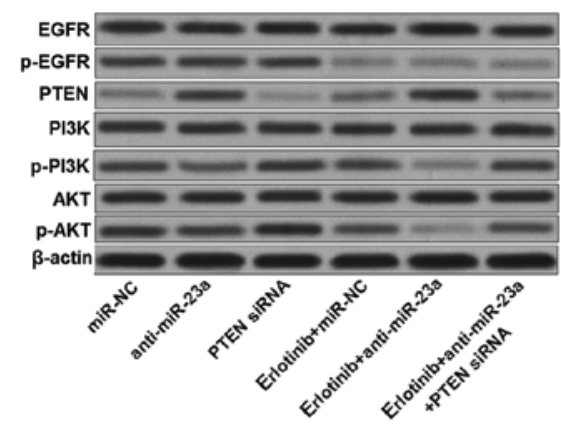

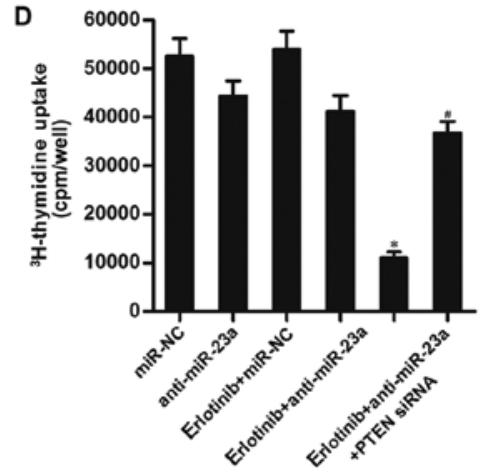

E
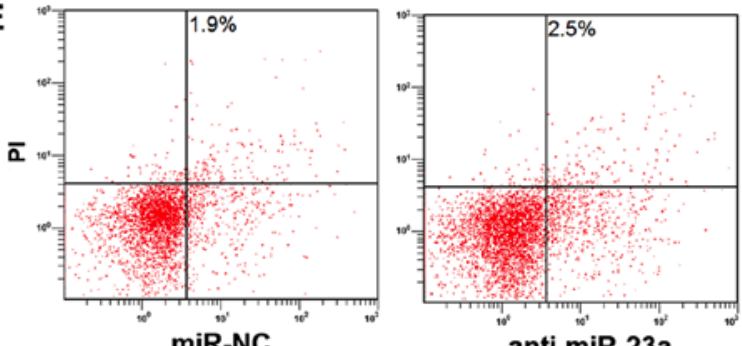

anti-miR-23a

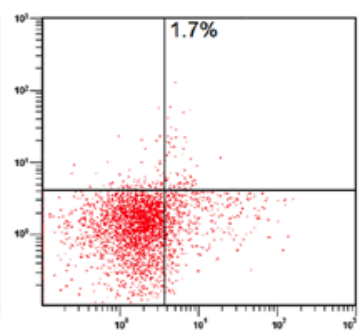

PTEN siRNA
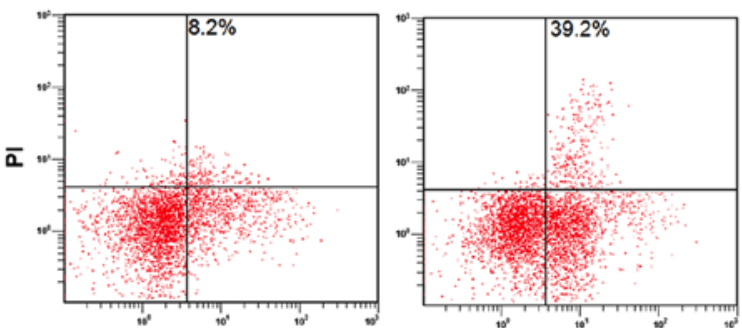

Erlotinib+anti-miR-23a

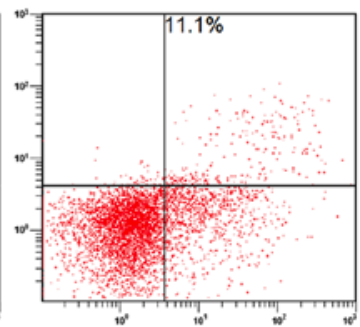

Erlotinib+anti-miR-23a

+PTEN SIRNA

Annexin V

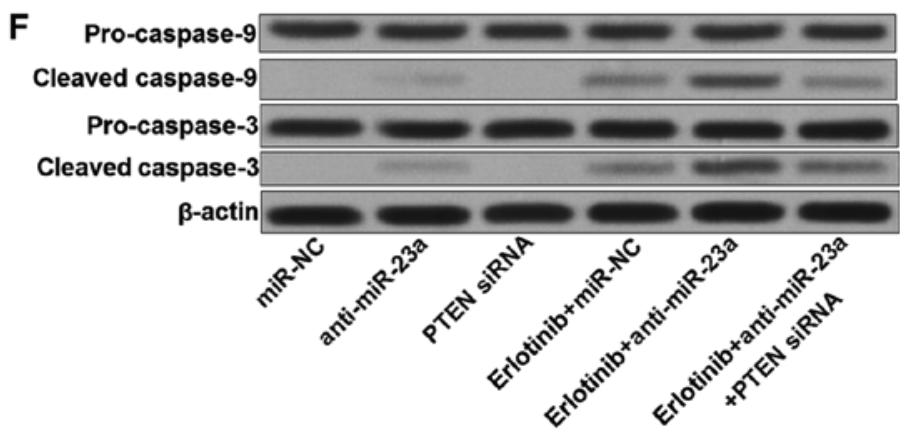

Figure 4. Anti-miR-23a resensitizes PC9 CSCs to erlotinib by upregulating the expression of PTEN. (A) Transfection efficiency of anti-miR-23a and PTEN siRNA on regulating the PTEN expression was detected by western blot analysis. (B) Effect of anti-miR-23a, PTEN siRNA and miR-23a on regulating the anti-tumor effect of erlotinib on PC9 CSCs. "P<0.05. (C) Expression of PTEN and phosphorylation of EGFR, PI3K and AKT were detected by western blot analysis after the PC9 CSCs were treated with anti-miR-23a, PTEN siRNA and erlotinib $(0.04 \mu \mathrm{M})$. (D) ${ }^{3} \mathrm{H}$-thymidine incorporation assays were performed to evaluate the effect of anti-miR-23a, PTEN siRNA and erlotinib on cell proliferation in PC9 CSCs. "P<0.05 vs. erlotinib+miR-NC group. ${ }^{~} \mathrm{P}<0.05$ vs. erlotinib+miR-NC group. (E) Flow cytometry was performed to measure apoptosis of PC9 CSCs treated with anti-miR-23a, PTEN siRNA and erlotinib. (F) Cleavage of caspase-9 and caspase-3 in PC9 CSCs was detected by western blot analysis after they were treated with anti-miR-23a, PTEN siRNA and erlotinib. 

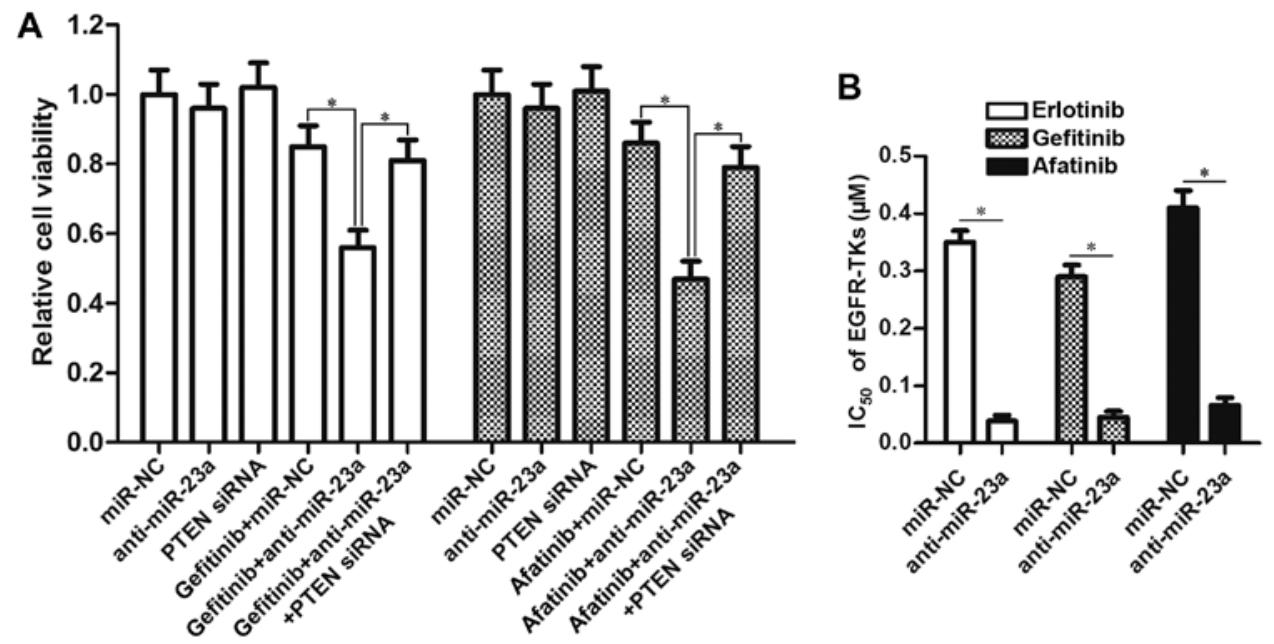

Figure 5. miR-23a antisense oligonucleotides enhance the antitumor effect of other EGFR-TKIs. (A) Anti-miR-23a promoted the cell death of PC9 CSCs induced by gefitinib $(0.04 \mu \mathrm{M})$ or afatinib $(0.04 \mu \mathrm{M}) .{ }^{*} \mathrm{P}<0.05$. (B) miR-23a antisense oligonucleotides decreased the $\mathrm{IC}_{50}$ of gefitinib, erlotinib and afatinib to PC9 CSCs. ${ }^{*} \mathrm{P}<0.05$

proved the synergistic effects of miR-23a antisense oligonucleotides on EGFR-TKIs. Mechanically, although erlotinib alone treatment inhibited the EGFR signaling, absence of PTEN in PC9 CSCs still induced the activation of PI3K and AKT. By contrast, combination with erlotinib and anti-miR23a inhibited the phosphorylation of EGFR, and upregulated the expression of PTEN. Phosphorylation of PI3K and AKT was significantly suppressed (Fig. 4C). As cell proliferation and apoptosis were regulated by PI3K/AKT pathway (22-24), we observed that combination with miR-23a antisense oligonucleotides and erlotinib induced significant inhibition of cell proliferation in PC9 CSCs (Fig. 4D). Moreover, antimiR-23a promoted erlotinib-induced apoptosis and cleavage of caspase- 9 and caspase- 3 by upregulating the expression of PTEN in PC9 CSCs (Fig. 4E and F). Taken together, we demonstrated that miR-23a antisense oligonucleotides resensitize lung cancer stem cells to erlotinib by increasing the expression of PTEN.

miR-23a antisense oligonucleotides enhance the antitumor effect of other EGFR-TKIs. To investigate the effect of anti-miR-23a on other EGFR-TKIs, we treated the PC9 CSCs with gefitinib and afatinib after transfection with anti-miR-23a and PTEN siRNA. The results of MTT demonstrated that knockdown of miR-23a also enhanced the antitumor effect of gefitinib and afatinib by upregulating the expression of PTEN (Fig. 5A). Intuitively, transfection with miR-23a antisense oligonucleotides significantly decreased the $\mathrm{IC}_{50}$ of all these EGFR-TKIs (gefitinib, erlotinib and afatinib) to PC9 CSCs (Fig. 5B). We therefore demonstrated the effect of miR-23a antisense oligonucleotides on resensitizing lung cancer stem cells to EGFR-TKIs.

\section{Discussion}

Approximately 25\% NSCLC patients exhibit mutation for epidermal growth factor receptor (EGFR). For these NSCLC patients, the cancer cells are sensitive to EGFR-TKIs such as erlotinib, gefitinib and afatinib. As the treatment of
EGFR-TKIs is the targeted molecular therapy without serious side-effects for NSCLC patients, EGFR-TKIs have become the first-line therapy for NSCLC $(4,5,25,26)$. However, almost all patients become resistant to EGFR-TKIs following 8-10 months of treatment $(27,28)$. Recent research has emphasized the importance of cancer stem cells (CSCs) for the acquired drug-resistance in cancers. Due to the high tumorigenicity and self-renewal, surviving CSCs in NSCLC inevitably become EGFR-TKIs resistant (29). In the present study, we found that the $\mathrm{IC}_{50}$ of erlotinib to PC9 CSCs was significantly higher than the PC9 non-CSCs. We demonstrated that lung cancer stem cells are insensitive to EGFR-TKIs.

MicroRNA-23a is reported to function as an oncogene in various human cancers. In NSCLC, high expression level of miR-23a shows poor prognosis (30). Furthermore, increasing evidence has declared the relationship between miRNAs and drug-resistance in cancer cells $(31,32)$. In the present study, we found that the expression level of miR-23a was significantly upregulated in PC9 CSCs rather than their corresponding non-CSCs. For reducing the drug-resistance of lung cancer stem cells to erlotinib, we knocked down the miR-23a by its antisense oligonucleotides in PC9 CSCs. Interestingly, we found the miR-23a antisense oligonucleotides can resensitize PC9 CSCs to erlotinib-induced cell death and apoptosis. Thus, for the first time, we reported the effect of anti-miR-23a on reversing the resistance of EGFR-TKIs by targeting lung cancer stem cells.

Phosphatase and tensin homologue (PTEN) is an important regulator for suppressing tumorigenesis and cancer development. PTEN mutation or loss induced uncontrolled cell cycle $(33,34)$. Among the pathways downstream of PTEN, PI3K/AKT whose phosphorylation is inhibited by PTEN regulates cell proliferation and apoptosis in cancer cells $(35,36)$. Recent studies demonstrate that expression of PTEN is regulated by certain miRNAs in cancers (37). In this study, we found that erlotinib failed to inhibit the phosphorylation of PI3K and AKT in PC9 CSCs. We indicate that this phenomenon is caused by the low-expression of PTEN in lung cancer stem cells. However, introduction with miR-23a 
antisense oligonucleotides increase the expression of PTEN in lung cancer stem cells. Therefore, the PC9 CSCs recover the sensitivity to erlotinib.

In conclusion, we demonstrated that lung cancer stem cells express low level of PTEN. As PTEN is targeted by miR-23a, we proved that introduction with miR-23a antisense oligonucleotides resensitize lung cancer stem cells to EGFR-TKIs by suppressing PI3K/AKT pathway. Combination of miR-23a antisense oligonucleotides and EGFR-TKIs could be attractive strategy for treatment of NSCLC.

\section{Acknowledgements}

Thanks are due to all the contributors who assisted with this study.

\section{References}

1. Siegel R, Naishadham D and Jemal A: Cancer statistics, 2013. CA Cancer J Clin 63: 11-30, 2013.

2. Allemani C, Weir HK, Carreira H, Harewood R, Spika D, Wang XS, Bannon F, Ahn JV, Johnson CJ, Bonaventure A, et al; CONCORD Working Group: Global surveillance of cancer survival 1995-2009: Analysis of individual data for 25,676,887 patients from 279 population-based registries in 67 countries (CONCORD-2). Lancet 385: 977-1010, 2015.

3. Sequist LV, Martins RG, Spigel D, Grunberg SM, Spira A, Jänne PA, Joshi VA, McCollum D, Evans TL, Muzikansky A et al: First-line gefitinib in patients with advanced non-small-cell lung cancer harboring somatic EGFR mutations. J Clin Oncol 26 : 2442-2449, 2008

4. Larsen JE and Minna JD: Molecular biology of lung cancer: Clinical implications. Clin Chest Med 32: 703-740, 2011.

5. Riely GJ, Pao W, Pham D, Li AR, Rizvi N, Venkatraman ES, Zakowski MF, Kris MG, Ladanyi M and Miller VA: Clinical course of patients with non-small cell lung cancer and epidermal growth factor receptor exon 19 and exon 21 mutations treated with gefitinib or erlotinib. Clin Cancer Res 12: 839-844, 2006.

6. Jiang J, Feng X, Zhou W, Wu Y and Yang Y: MiR-128 reverses the gefitinib resistance of the lung cancer stem cells by inhibiting the c-met/PI3K/AKT pathway. Oncotarget 7: 73188-73199, 2016.

7. Sharma SV, Lee DY, Li B, Quinlan MP, Takahashi F, Maheswaran S, McDermott U, Azizian N, Zou L, Fischbach MA, et al: A chromatin-mediated reversible drug-tolerant state in cancer cell subpopulations. Cell 141: 69-80, 2010.

8. Dean M, Fojo T and Bates S: Tumour stem cells and drug resistance. Nat Rev Cancer 5: 275-284, 2005.

9. Kobayashi I, Takahashi F, Nurwidya F, Nara T, Hashimoto M, Murakami A, Yagishita S, Tajima K, Hidayat M, Shimada N, et al: Oct4 plays a crucial role in the maintenance of gefitinib-resistant lung cancer stem cells. Biochem Biophys Res Commun 473: 125-132, 2016.

10. Bartel DP: MicroRNAs: Target recognition and regulatory functions. Cell 136: 215-233, 2009.

11. Gargalionis AN and Basdra EK: Insights in microRNAs biology. Curr Top Med Chem 13: 1493-1502, 2013.

12. Sun Y,He N, Dong Y and Jiang C: MiR-24-BIM-Smac/DIABLO axis controls the sensitivity to doxorubicin treatment in osteosarcoma. Sci Rep 6: 34238, 2016.

13. Ye Z, Hao R, Cai Y, Wang X and Huang G: Knockdown of miR-221 promotes the cisplatin-inducing apoptosis by targeting the BIM-Bax/Bak axis in breast cancer. Tumour Biol 37 4509-4515, 2016

14. Chai S, Ng KY, Tong M, Lau EY, Lee TK, Chan KW, Yuan YF, Cheung TT, Cheung ST, Wang XQ, et al: Octamer 4/microRNA1246 signaling axis drives Wnt/ $\beta$-catenin activation in liver cancer stem cells. Hepatology 64: 2062-2076, 2016.

15. Wang H, Meng Y, Cui Q, Qin F, Yang H, Chen Y, Cheng Y, Shi J and Guo Y: MiR-101 targets the EZH2/Wnt/ $\beta$-catenin the pathway to promote the osteogenic differentiation of human bone marrow-derived mesenchymal stem cells. Sci Rep 6: 36988, 2016.

16. Feng X, Jiang J, Shi S, Xie H, Zhou L and Zheng S: Knockdown of miR-25 increases the sensitivity of liver cancer stem cells to TRAIL-induced apoptosis via PTEN/PI3K/Akt/Bad signaling pathway. Int J Oncol 49: 2600-2610, 2016.
17. Lee SO, Yang X, Duan S, Tsai Y, Strojny LR, Keng P and Chen Y: IL-6 promotes growth and epithelial-mesenchymal transition of $\mathrm{CD}_{133^{+}}$cells of non-small cell lung cancer. Oncotarget 7: 6626-6638, 2016

18. French R, Hayward O, Jones S, Yang W and Clarkson R: Cytoplasmic levels of cFLIP determine a broad susceptibility of breast cancer stem/progenitor-like cells to TRAIL. Mol Cancer 14: 209, 2015.

19. Livak KJ and Schmittgen TD: Analysis of relative gene expression data using real-time quantitative PCR and the 2(-Delta Delta C(T)) method. Methods 25: 402-408, 2001.

20. Murakami A, Takahashi F, Nurwidya F, Kobayashi I, Minakata K, Hashimoto M, Nara T, Kato M, Tajima K, Shimada N, et al: Hypoxia increases gefitinib-resistant lung cancer stem cells through the activation of insulin-like growth factor 1 receptor. PLoS One 9: e86459, 2014

21. Gadgeel SM and Wozniak A: Preclinical rationale for PI3K/Akt/ mTOR pathway inhibitors as therapy for epidermal growth factor receptor inhibitor-resistant non-small-cell lung cancer. Clin Lung Cancer 14: 322-332, 2013.

22. Wang T, Gong X, Jiang R, Li H, Du W and Kuang G: Ferulic acid inhibits proliferation and promotes apoptosis via blockage of PI3K/Akt pathway in osteosarcoma cell. Am J Transl Res 8: 968-980, 2016

23. Wang R, Zhang Q, Peng X, Zhou C, Zhong Y, Chen X, Qiu Y, Jin M, Gong M and Kong D: Stellettin B induces G1 arrest, apoptosis and autophagy in human non-small cell lung cancer A549 cells via blocking PI3K/Akt/mTOR pathway. Sci Rep 6: 27071, 2016.

24. Wu YR, Qi HJ, Deng DF, Luo YY and Yang SL: MicroRNA-21 promotes cell proliferation, migration, and resistance to apoptosis through PTEN/PI3K/AKT signaling pathway in esophageal cancer. Tumour Biol 37: 12061-12070, 2016.

25. Sharma SV, Bell DW, Settleman J and Haber DA: Epidermal growth factor receptor mutations in lung cancer. Nat Rev Cancer 7: 169-181, 2007.

26. Yu HA and Pao W: Targeted therapies: Afatinib - new therapy option for EGFR-mutant lung cancer. Nat Rev Clin Oncol 10: 551-552, 2013.

27. Engelman JA, Zejnullahu K, Mitsudomi T, Song Y, Hyland C, Park JO, Lindeman N, Gale CM, Zhao X, Christensen J, et al: MET amplification leads to gefitinib resistance in lung cancer by activating ERBB3 signaling. Science 316: 1039-1043, 2007.

28. Chong CR and Jänne PA: The quest to overcome resistance to EGFR-targeted therapies in cancer. Nat Med 19: 1389-1400, 2013.

29. Murakami A, Takahashi F, Nurwidya F, Kobayashi I, Minakata K, Hashimoto M, Nara T, Kato M, Tajima K, Shimada N, et al: Hypoxia increases gefitinib-resistant lung cancer stem cells through the activation of insulin-like growth factor 1 receptor. PLoS One 9: e86459, 2014.

30. Qu WQ, Liu L and Yu Z: Clinical value of microRNA-23a upregulation in non-small cell lung cancer. Int J Clin Exp Med 8: 13598-13603, 2015.

31. Zhou S, Huang Q, Zheng S, Lin K, You J and Zhang X: miR-27a regulates the sensitivity of breast cancer cells to cisplatin treatment via BAK-SMAC/DIABLO-XIAP axis. Tumour Biol 37: 6837-6845, 2016

32. Yin W, Nie Y, Zhang Z, Xie L and He X: miR-193b acts as a cisplatin sensitizer via the caspase-3-dependent pathway in HCC chemotherapy. Oncol Rep 34: 368-374, 2015.

33. Wang YS, Wang YH, Xia HP, Zhou SW, Schmid-Bindert G and Zhou CC: MicroRNA-214 regulates the acquired resistance to gefitinib via the PTEN/AKT pathway in EGFR-mutant cell lines. Asian Pac J Cancer Prev 13: 255-260, 2012.

34. Darido C, Georgy SR, Wilanowski T, Dworkin S, Auden A, Zhao Q, Rank G, Srivastava S, Finlay MJ, Papenfuss AT, et al: Targeting of the tumor suppressor GRHL3 by a miR21-dependent proto-oncogenic network results in PTEN loss and tumorigenesis. Cancer Cell 20: 635-648, 2011.

35. Carnero A, Blanco-Aparicio C, Renner O, Link W and Leal JF: The PTEN/PI3K/AKT signalling pathway in cancer, therapeutic implications. Curr Cancer Drug Targets 8: 187-198, 2008.

36. Ma Y, Zhang P, Gao Y, Fan H, Zhang M and Wu J: Evaluation of AKT phosphorylation and PTEN loss and their correlation with the resistance of rituximab in DLBCL. Int J Clin Exp Pathol 8: 14875-14884, 2015

37. Wang J, Xu J, Fu J, Yuan D, Guo F, Zhou C and Shao C: MiR-29a regulates radiosensitivity in human intestinal cells by targeting PTEN gene. Radiat Res 186: 292-301, 2016. 